\title{
Caracterización de la heterogeneidad estructural en polipropileno polimórfico mediante espectroscopia vibracional: microscopia IR y Raman
}

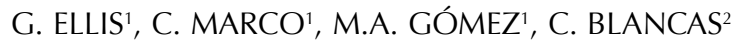 \\ ${ }^{1}$ Instituto de Ciencia y Tecnología de Polímeros, CSIC, Juan de la Cierva 3, 28006 Madrid, España. \\ ${ }^{2}$ Centro Tecnológico de Repsol YPF, Carretera de Extremadura Km 18, 28931 Móstoles, Madrid
}

\begin{abstract}
Una serie de muestras polimórficas de polipropileno isotáctico, obtenidos por nucleación selectiva, fueron estudiadas mediante espectroscopia vibracional. Se observó una correlación entre los espectros obtenidos y el parámetro de Turner-Jones, característico de la fracción del polimorfo $\beta$. Se evaluaron aspectos de la heterogeneidad estructural utilizando técnicas de mapeo con microscopia IR, microscopia Raman confocal, microscopia IR con radiación sincrotrón y microscopia IR de imagen.
\end{abstract}

Palabras clave: polipropileno, polimorfismo, heterogeneidad, microscopia vibracional, IR, Raman

\section{Caracterization of structural heterogeneity in polymorphic polypropylene by vibrational spectroscopy: IR and Raman microscopy}

A series of polymorphic samples of isotactic polypropylene, obtained via selective nucleation, were studied by vibrational spectroscopy. A correlation was observed between the spectra obtained and the Turner-Jones parameter, characteristic of the fraction of the $\beta$ polymorph. Aspects of the structural heterogeneity in the samples were evaluated using mapping techniques with IR micrsocopy, confocal Raman microscopy, synchrotron IR microscopy and IR imaging microscopy.

Keywords: polypropylene, polymorphism, heterogeneity, vibrational microscopy, IR, Raman

\section{INTRODUCCIÓN}

En la actualidad existe un gran interés en el diseño de materiales poliméricos basados en polipropileno isotáctico, iPP, particularmente dirigido hacía su comportamiento de cristalización y los fenómenos de nucleación, así como su correlación con propiedades mecánicas, ópticas y superficiales de gran importancia tecnológica. Es conocido que el iPP cristaliza en cuatro polimorfos diferentes, siendo la modificación monoclínica $(\alpha)$ la predominante, la cual se genera en las condiciones convencionales de proceso. Sin embargo, la aparición conjunta con la modificación trigonal $(\beta)$ es frecuente, provocando cambios importantes en las propiedades físicas del material [1]. Se han descrito diferentes estrategias para alcanzar altas concentraciones de la forma $\beta$ en el iPP. Recientemente se han desarrollado agentes nucleantes específicos inductores de este polimorfo con el fin de diseñar tanto las propiedades ópticas como mecánicas del polímero. En este sentido, la caracterización de iPP polimórfico es fundamental, lo que habitualmente se realiza mediante calorimetría diferencial de barrido (DSC), microscopia óptica de luz polarizada (POM), y difracción de rayos-X (WAXD). Esta última técnica es fundamental para la determinación del contenido $\beta$ en iPP, y para este fin, desde hace más de 40 años se emplea el parámetro $k$ desarrollado por Turner-Jones [2].

Recientemente el estudio por espectroscopia vibracional de muestras de iPP con altos contenidos en la modificación trigonal ha permitido diferenciar entre los polimorfos $\alpha$ y $\beta$ utilizando la microscopia IR [3]. Con el fin de comprobar el potencial de la espectroscopia vibracional para el estudio de iPP polimórfico, se han examinado una serie de muestras polimórficas de iPP, utilizando técnicas de microscopia vibracional de última generación, destacando en particular la microscopia IR por radiación sincrotrón, la microscopia IR de imagen mediante un detector bidimensional tipo FPA, y la microscopia Raman confocal.

\section{EXPERIMENTAL}

\subsection{Materiales}

Tres tipos de polipropileno isotáctico fueron proporcionados por REPSOL-YPF. Dos de ellos fueron grados comerciales de reactor de- nominados $P 1$ (peso molecular promedio viscoso, $\mathrm{Mv}=164,700$ [4]) y $P 2(\mathrm{Mv}=116,200$, índice de polidispersidad, I.P. $=4)$, y el tercero, un grado de reología controlada, denominado $P 3(\mathrm{Mv}=100,000$, I.P. = 2,6) [5]. Se emplearon 3 agentes nucleantes de diferente naturaleza; 1,3: 2,4-bis-(3,4-dimetilbenziliden sorbitol), MILLAD 3988 de Milliken Chemical, denominado $n 1, \mathrm{~N}, \mathrm{~N}^{\prime}$-diciclohexíl-2,6-naftalén dicarboxamida, NJ-STAR NU100 de NJC-Rika, denominado $n 2$, y ácido pimélico, denominado $n 3$. Una serie de materiales polimórficos, $P 1+n 1$ y $P 1+n 2$, fueron preparados a concentraciones de agente nucleante entre 0,025 $-1,0 \%$ en peso, mediante mezclado en una extrusora de doble husillo utilizando las condiciones descritos en un trabajo anterior [4]. Otra serie de muestras, $P 2+n 3$ y $P 3+n 3$, fueron preparadas a partir de una disolución $0,25 \mathrm{M}$ en $\mathrm{CHCl}_{3}$ a concentraciones entre $0,025-1,0 \%$ en peso de $n 3$, utilizando un método similar al descrito por Wang [6]. Se prepararon películas delgadas mediante compresión a partir del fundido, y posteriormente fueron sometidas a una fusión previa entre portas de microscopio para eliminar la historia térmica [4], y cristalizadas a distintas velocidades de enfriamiento, en una célula de temperatura Mettler FP80T acoplada a un microscopio Reichert Zetopan-Pol de luz polarizada. En último término su comportamiento térmico y morfología fue analizado por DSC, WAXD y análisis termoóptico [7-9].

\subsection{Técnicas experimentales}

Los espectros IR y Raman convencionales fueron registrados en un sistema Perkin-Elmer Spectrum GX2000 FT- IR y 2000R FT-Raman, respectivamente, utilizando una resolución espectral de $4 \mathrm{~cm}^{-1}$ [10].

Las medidas por microscopia IR fueron realizadas en un microscopio Perkin-Elmer i-Series IMAGE con detector MCT acoplado al espectrómetro GX2000 (denominado sistema M1) [10], y un Perkin-Elmer Spectrum Spotlight 300 [11] incorporando un detector bidimensional, 
acoplado a un espectrómetro FT-IR Spectrum One (sistema M2). Ambos equipos poseen plataformas digitales programables a través del software de control que permite obtener mapas espectroscópicos bidimensionales, y polarizadores visibles para distinguir entre las esferulitas en iPP cristalino, como en un microscopio óptico de luz polarizada.

Las medidas de microscopia IR por radiación sincrotrón fueron realizadas en la línea MIRAGE, del anillo SuperACO, de LURE, en Paris, Francia, empleando un microscopio IR Nic-Plan acoplado a un Magna 560 FTIR de Nicolet, equipado con una plataforma digital (sistema M3). Detalles de la técnica, el equipo y su acoplamiento al anillo de almacenamiento se puede encontrar en las referencias [12,13].

Los mapas de Raman fueron obtenidos con un equipo RM1000 microscopio Raman confocal de Renishaw (sistema M4) [14], con una fuente de excitación láser de $\mathrm{Ar}^{+}$a 514,5 nm, con una potencia de $4 \mathrm{~mW}$ enfocado en la muestra por un objetivo 50x N-plan de Leica, empleándose un tiempo de adquisición de 20s para cada punto de medida.

Los difractogramas de rayos- $X$ fueron realizados mediante el uso de la radiación sincrotrón en la Polymer Beamline, de HASYLAB, en DESY Hamburgo, Alemania [8].

\section{RESULTADOS Y DISCUSIÓN}

El iPP no nucleado, o con el agente $n 1$ cristaliza predominantemente en la forma monoclínica. Sin embargo, los nucleantes $n 2$ y $n 3$ inducen la cristalización de la forma trigonal [5,8]. Las variaciones en los difractogramas de rayos-X y en los espectros IR y Raman en función del tipo de agente se muestran en la Figura 1. En todos los casos se puede diferenciar entre los polimorfos $\alpha$ y $\beta$, pero mientras estas son evidentes por WAXD, por espectroscopia son considerablemente más sutiles. A partir de los difractogramas, y mediante la relación descrita por Turner-Jones [2] se puede calcular el parámetro $k$ relacionado con el contenido del polimorfo trigonal en una muestra. Se ha demostrado que $k$ aumenta con la concentración de agente nucleante de la forma $\beta$ para $n 2$ [8], hecho que ocurre también cuando se utiliza el nucleante $n 3$ [5]. En los espectros vibracionales, varias bandas son sensibles a la variación de la fracción del polimorfo trigonal. La figura 2 muestra como ejemplo la correlación entre $k$ y la relación de las bandas a 1296 y $1305 \mathrm{~cm}^{-1}$ para muestras de $P 2$ y $P 3$ nucleadas con varias concentraciones de $n 3$, y enfriadas desde el fundido a $20^{\circ} \mathrm{C} . \mathrm{min}^{-1}$, a partir de los espectros registrados en el sistema $M 1$ con una apertura de $100 \mu \mathrm{m}$. Se ha observado que tanto la concentración del agente como las condiciones de cristalización ejercen una importante influencia sobre la correlación ya que, por la naturaleza compleja de los modos vibracionales del iPP, el espectro de polipropileno es sensible tanto a cambios en la concentración de los polimorfos, como en el orden o perfección cristalina, estando ambos fenómenos solapados. Para iguales condiciones de cristalización, los datos obtenidos a partir de estas correlaciones son comparables, lo que ha permitido obtener información sobre la heterogeneidad en función de la concentración de agente [15], y de la matriz de iPP. En este último caso y en iguales condiciones de cristalización y concentración se ha observado que la nucleación de la forma $\beta$ es más eficiente en el polímero de reactor, $P 2$ que en el polímero obtenido por escisión, $P 3[5,9]$.

Evidentemente, el estudio de la heterogeneidad de estos sistemas depende de la resolución espacial empleada en las medidas, ya que el tamaño de los dominios cristalinos está condicionado por la distribución de núcleos, y por la velocidad de cristalización. Enfriando a una velocidad inferior, $2^{\circ} \mathrm{C} . \mathrm{min}^{-1}$, la correlación descrita en la Figura 2 no solamente cambia de pendiente, sino que además está perturbada por el aumento de la heterogeneidad del sistema, la cual es importante a esta resolución espacial.
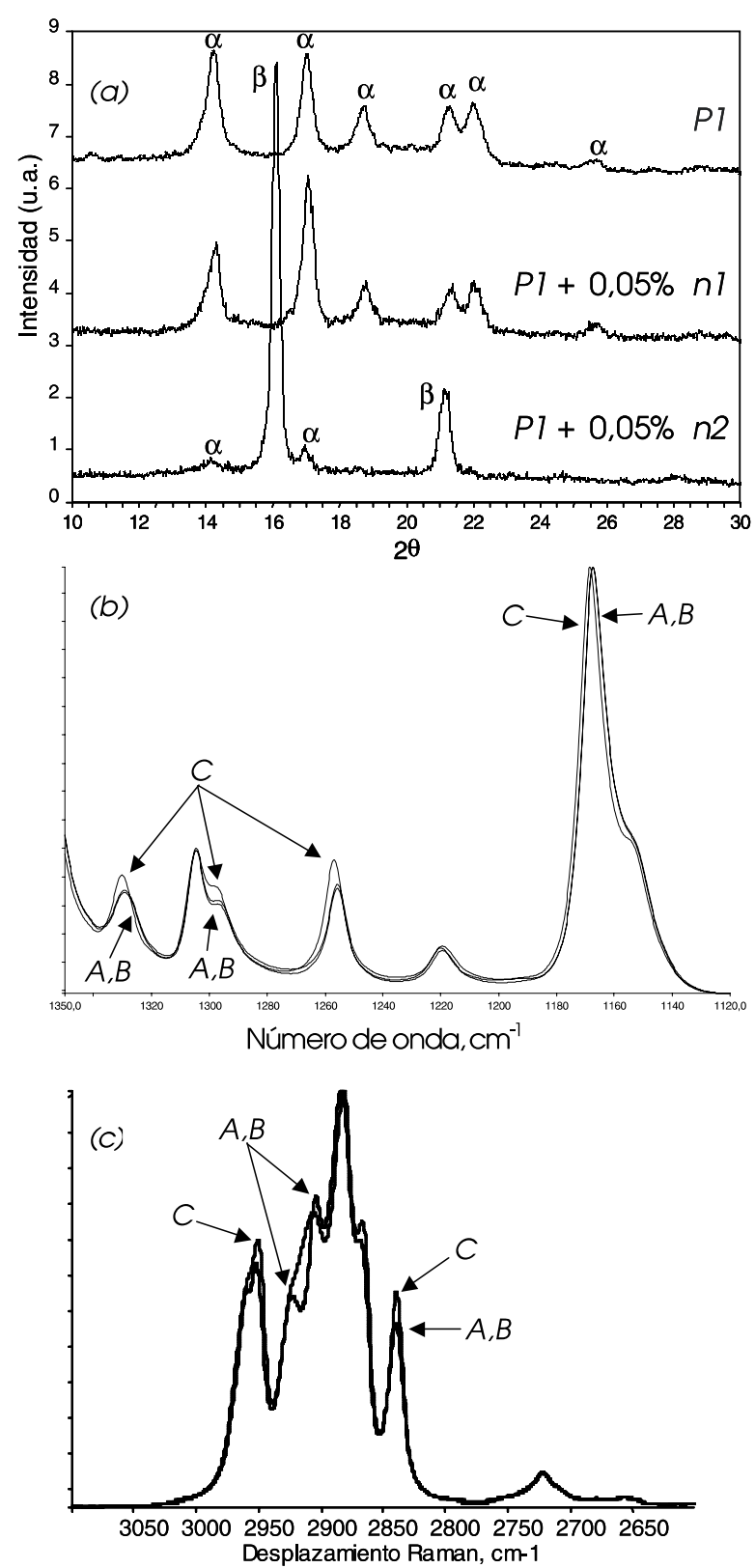

Fig. 1 (a) Difractogramas de rayos-X, (b) espectros FT-IR, y (c) espectros FT-Raman de iPP cristalizados a $10^{\circ} \mathrm{C} \cdot \mathrm{min}^{-1}$. A $=P 1, \mathrm{~B}=P 1+$ $0,05 \% n 1, \mathrm{C}=P 1+0,05 \% n 2$.

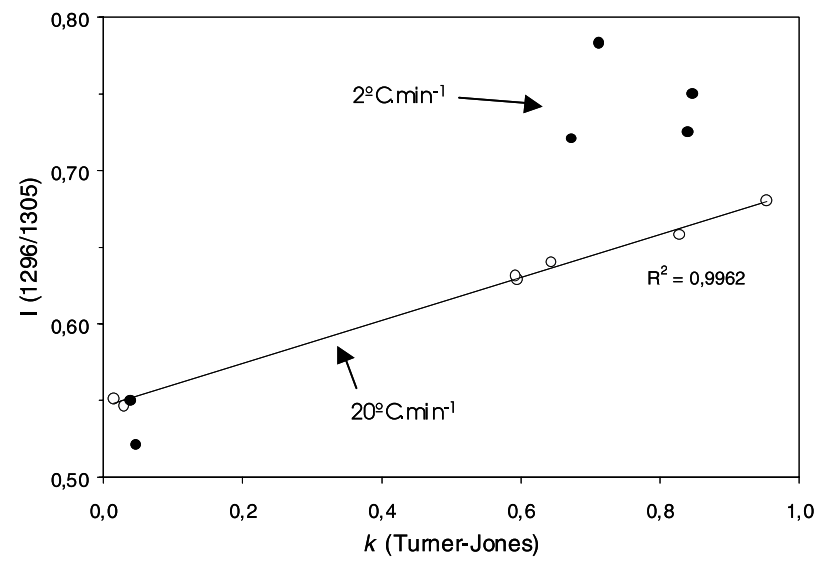

Fig. 2 Relación de bandas del espectro IR frente al parámetro $k$ para iPP nucleado con ácido pimélico, $n 3$ 
Reduciendo la resolución espacial, por ejemplo, mediante espectroscopia IR convencional, se obtiene un promedio sobre un área mayor, y el efecto de la heterogeneidad tiene menos importancia. Sin embargo, aumentando la resolución espacial, se puede obtener información mucho más detallada sobre los dominios y su distribución. De esta forma, a una resolución espacial de $25 \mu \mathrm{m}$ se pueden observar diferencias importantes en la naturaleza de la heterogeneidad en función de la concentración de agente y tipo de matriz, Figura 3, utilizando como ejemplo la relación de bandas $1256 \mathrm{~cm}^{-1} / 1168 \mathrm{~cm}^{-1}$. Sin embargo, para mayor resolución espacial, es necesario una mayor reducción de la apertura del microscopio, con la correspondiente disminución en la energía disponible, y en la relación señal/ruido, lo que aumenta de forma prohibitiva en el tiempo de medida para obtener mapas representativos de la muestra, aún en el caso de que ello sea posible.

La radiación procedente del anillo de almacenamiento de un sincrotrón es una fuente de energía ideal para la microscopia IR, debido a la alta luminosidad y a la coherencia del haz, y la distribución espectral continua en todo el intervalo espectroscópico [12]. La relación señal/ruido para aperturas pequeñas (menor de $10 \mu \mathrm{m}$ ), es más de 100 veces mayor que para una fuente IR convencional, lo que permite obtener espectros de alta calidad a una resolución espacial de hasta 3 x $3 \mu \mathrm{m}[16]$.

La Figura 4(a) presenta una microfotografía de luz polarizada de una esferulita trigonal en un campo de esferulitas monoclínicas de iPP que se ha generado de forma espontánea durante la cristalización isoterma a $130^{\circ} \mathrm{C}$ de una muestra de iPP no nucleado [3]. Las Figuras 4(b) y 4(c) representan mapas estructurales de las zonas indicados en la fotografía, construidos a partir de los espectros micro-IR y microRaman, respectivamente.

En la Figura 4(b), se han empleado dos relaciones de bandas, seleccionadas en función de la información estructural de interés. La relación de bandas $1296 \mathrm{~cm}^{-1} / 1305 \mathrm{~cm}^{-1}$ corresponde a diferencias prin- cipalmente debidas al polimorfismo, mapa 1, y se puede distinguir claramente entre la esferulita de la forma $\beta$ y sus vecinos de la forma $\alpha$. La relación de bandas $998 \mathrm{~cm}^{-1} / 973 \mathrm{~cm}^{-1}$, mapa 2, es sensible a la cristalinidad del iPP, pudiéndose apreciar que la zona derecha del mapa 2, de mayor claridad, corresponde a un material de mayor cristalinidad, de acuerdo con la mayor densidad de los cristales monoclínicos.

Del mismo modo, en la Figura 4(c), se muestran los mapas obtenidos por microscopia Raman confocal de un área de 50 x $34 \mu \mathrm{m}$ marcado en la Figura 4(a), y empleando una alta resolución espacial de $1 \mu \mathrm{m}$. Los mapas se han generado utilizando, por una parte, las variaciones en la intensidad de la banda alrededor de $2920 \mathrm{~cm}^{-1}$ asociada a la tensión asimétrica del grupo metileno y, por otra, utilizando la anchura total a media altura (FWHM) de la misma banda. Se observa que ambos tipos de esferulita se pueden diferenciar perfectamente, como en el caso de la microscopia IR.. Sin embargo, aunque las diferencias en los mapas Raman son espectaculares, cabe mencionar que para construir un mapa tan grande en el modo confocal fue necesario registrar 1700 espectros durante $9 \mathrm{~h}$. El hecho de que la banda de interés esté solapada con otras bandas vibracionales, como se puede apreciar en la Figura 1c, significa que es necesario utilizar la deconvolución espectral y ajuste de curvas para obtener la intensidad (área) y FWHM de la banda a $2920 \mathrm{~cm}^{-1}$ en cada punto y, posteriormente, construir los mapas. Por este motivo, se ha empleado un tiempo de adquisición de $20 \mathrm{~s}$ para cada punto, y obteniéndose así una relación señal-ruido óptima en cada espectro, lo que introduce menos errores en el ajuste de curvas. En ejemplos de sistemas heterogéneos donde la banda de interés está aislada de otras bandas, se puede reducir dramáticamente el tiempo de adquisición en el modo confocal y, es posible, obtener imágenes Raman en pocos minutos utilizando el modo de imagen global [14].

Por último, la Figura 5 muestra el mapa correspondiente a la relación de bandas $1296 \mathrm{~cm}^{-1} / 1305 \mathrm{~cm}^{-1}$ sobre un área de $431 \mu \mathrm{m} \times 419 \mu \mathrm{m}$ de la misma muestra de la figura 4(a), registrado en un microscopio FT-IR de
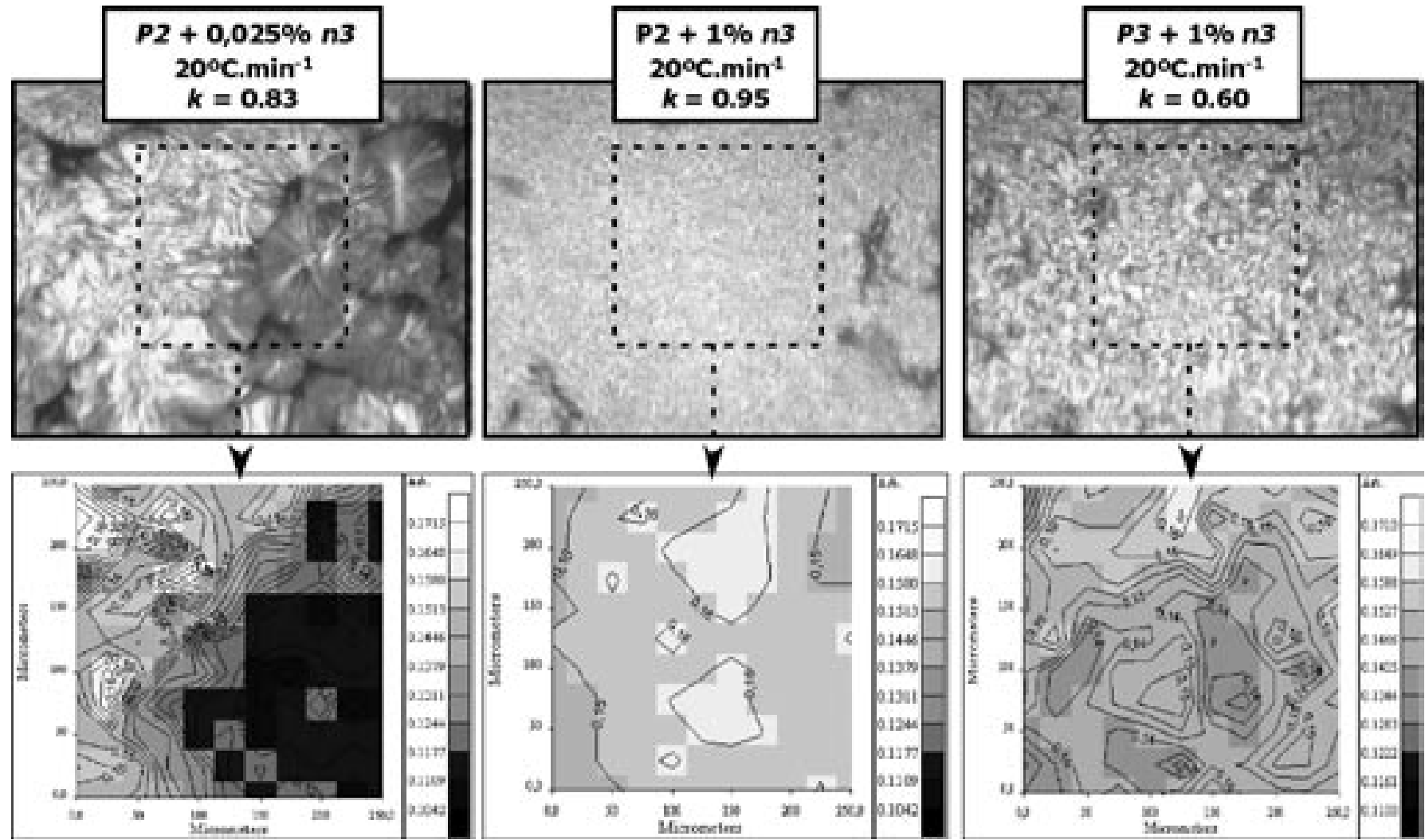

Fig. 3. Microfotografías y los correspondientes mapas polimórficos de muestras nucleadas de iPP en función de la concentración de agente nucleante, y la velocidad de enfriamiento. Los colores claras muestran más alta contenido en la forma $\beta$, y las oscuras más altas en la forma $\alpha$. 

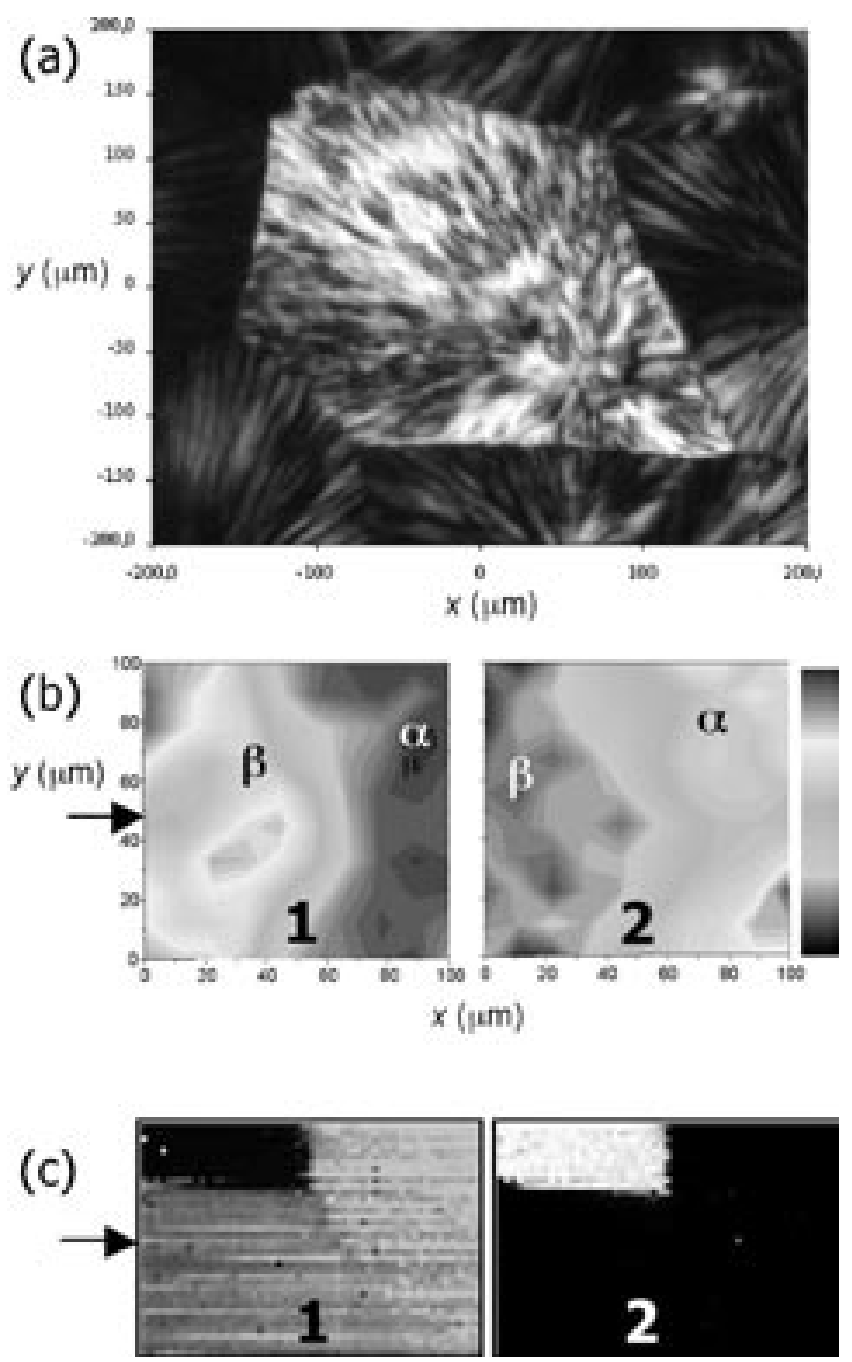

Fig. 4. Mapas con alta resolución espacial; (a) microscopia óptica de luz polarizada, (b) microscopia IR con radiación sincrotrón (apertura de $6 \times 6 \mu \mathrm{m}$, registrando un área de $100 \times 100 \mu \mathrm{m}$ en aproximadamente 2 horas); mapa 1, relación de bandas $1296 \mathrm{~cm}^{-1} / 1305 \mathrm{~cm}^{-1}$ (mapa de polimorfos), y mapa 2; relación de bandas $998 \mathrm{~cm}^{-1} / 973 \mathrm{~cm}^{-1}$ (mapa de cristalinidad), y (c) microscopia Raman confocal; mapa 1, intensidad, y mapa 2, FWHM, de la banda a $2920 \mathrm{~cm}^{-1}$

imagen, $M 2$. En este equipo, mediante un detector tipo bidimensional y una óptica de magnificación de imagen, se consigue una resolución espacial de la imagen en el plano focal que corresponde a 6,25 $\mu \mathrm{m}$ [11]. El tiempo de adquisición de la imagen IR en la Figura 5 fue aproximadamente de 50 minutos. La gran ventaja de este sistema es que permite obtener mapas relativamente grandes, en este caso un mapa estructural sensible al tipo de polimorfo, de toda la esferulita y sus vecinas con espectros individuales de alta calidad, como se muestra en la Figura 5 por los espectros correspondientes a los puntos indicados en el imagen.

La gran velocidad de este sistema ha permitido registrar mapas de muestras polimórficas en función de la temperatura. En la Figura 6 se observan una serie de mapas correspondientes a un área fija de $450 \mu \mathrm{m} \times 568 \mu \mathrm{m}$ de la muestra $P 2+0,025 \%$ n3. La muestra, montada entre ventanas de BrK, fue calentada in situ hasta diferentes temperaturas en una célula de alta temperatura para microscopia IR (CIC Photonics). A través de la relación $1296 \mathrm{~cm}^{-1} / 1305 \mathrm{~cm}^{-1}$, se monitorizó la distribución de polimorfos antes y después de la transición polimórfica $\beta-\alpha$, que ocurre alrededor de los $155^{\circ} \mathrm{C}$ [8]. El mapa en la Figura 6(a) corresponde a temperatura ambiente, donde las zonas más claras

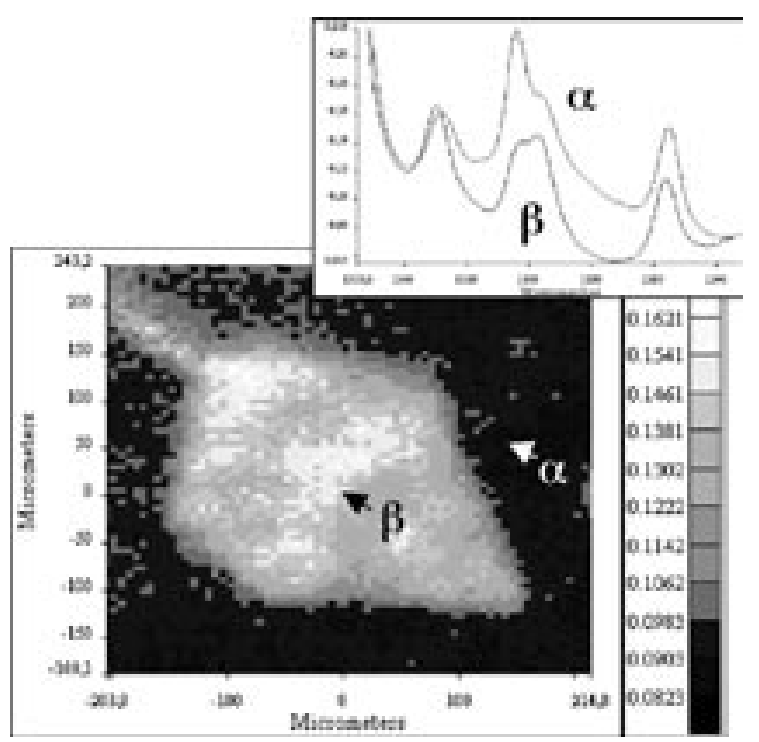

Fig. 5. Mapa correspondiente a la figura 4(b) por un sistema FT-IR de imagen, y dos espectros correspondientes a las posiciones marcadas.
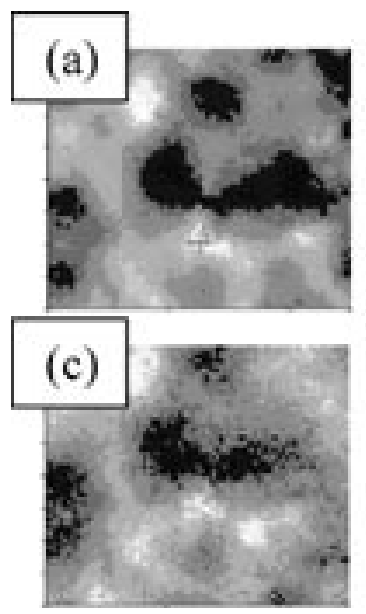

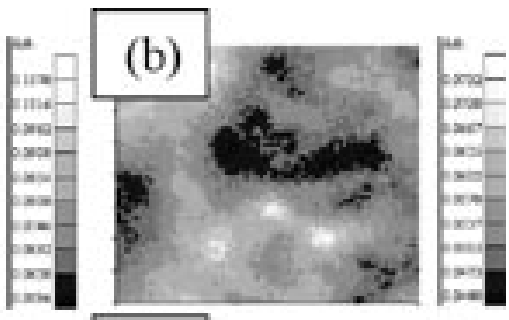

(d)
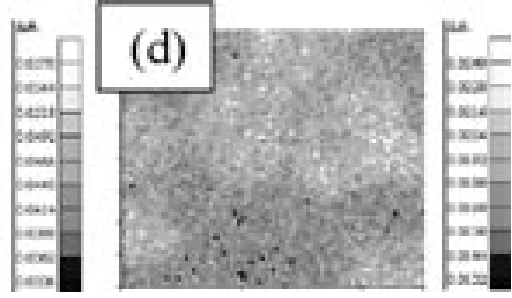

Fig. 6 Variaciones en la distribución de polimorfos en iPP, $P 2+$ $0,025 \% n 3$, reflejadas en los mapas de heterogeneidad obtenidos por microscopia IR de imagen en función de la temperatura (a) 25, (b) 90, (c) $120 \mathrm{y}(\mathrm{d}) 160^{\circ} \mathrm{C}$.

están asociadas con el $\beta$-iPP. A medida que se aumenta la temperatura, la distribución de los mapas varía hasta después de la transición $\beta-\alpha$, donde el mapa se observa más homogéneo.

\section{CONCLUSIONES}

El uso de la espectroscopia vibracional ha permitido establecer correlaciones entre la fracción de la forma trigonal en muestras de iPP polimórfico, dada por el parámetro $k$ de Turner-Jones, cuando las condiciones de cristalización están definidas. Estas correlaciones están afectadas por la velocidad de cristalización y por la relación entre el tamaño de los dominios y la resolución espacial empleada en las medidas. Se ha demostrado que la aplicación de la microscopia Raman confocal y la microscopia IR con radiación sincrotrón es posible obtener mapas estructurales de iPP polimórfico a una alta resolución espacial. Por otra parte, mediante el uso de la microscopia IR de imagen es posible obtener datos muy detallados sobre la heterogeneidad estructural en áreas representativas de la muestra en tiempos razonables. 


\section{AGRADECIMIENTOS}

Este estudio ha sido financiado por la CICYT, MAT-98-0194. Agradecemos a la Comunidad Europea "Enhancing Access to Research Infrastructures", Proyecto IM008-02 para acceso al sincrotrón de LURE, así como Prof. Paul Dumas por su ayuda. Acceso al sincrotrón de DESY fue subvencionado por la EU en el Proyecto IHP-HRRICT-1999-00040. Finalmente, nuestros agradecimientos al Dr. Robert Alexander y Perkin-Elmer Ltd, Seer Green, UK por su ayuda y el uso del Spotlight Sistema de Imagen FT-IR, y al Dr. Tim Smith y Renishaw plc, Wotton-under-Edge, UK por su ayuda y el uso del microscopio Raman confocal.

\section{BIBLIOGRAFÍA}

1. J. Varga, "Crystallization, melting and supermolecular structure of isotactic polypropylene", pp. 56-115, en Polypropylene. Structure, blends and composites. Vol.1. Structure and Morphology, Ed. J. Karger-Kocsis, Chapman \& Hall, London (UK) 1995

2. A. Turner-Jones, J. M. Aizlewood, D. R. Beckett. "Crystalline forms of isotactic polypropylene". Makromol. Chem., 75, 134-158 (1964)

3. G. Ellis, C. Marco, M. A. Gómez. "Mapping the crystalline morphology of isotactic polypropylene by infrared microscopy". Int. J. Vibr. Spectrosc. [www.ijvs.com], 5[4], 7 (2001)

4. C. Marco, M.A. Gómez, G. Ellis, J.M. Arribas. “Highly efficient nucleating additive for isotactic polypropylene studied by differential scanning calorimetry". J. Appl. Polym. Sci., 84[9], 1669-1679 (2002)
5. C. Blancas "Propiedades en estado sólido de polipropilenos de reología controlada", Tesis Doctoral, Universidad Complutense de Madrid (2002)

6. K. Wang, K. Mai, H. Zeng. "Isothermal crystallization behavior and melting characteristics of injection sample of nucleated polypropylene". J. Appl. Polym. Sci., 78[14], 2547 (2000)

7. C. Marco, G. Ellis, M.A. Gómez, J.M. Arribas, “Comparative study of the nucleation activity of third generation sorbitol-based nucleating agents for isotactic polypropylene". J. Appl. Polym. Sci., 84[13], 2440-2450 (2002)

8. C. Marco, M.A. Gómez, G. Ellis, J.M. Arribas. “Activity of a $\beta$-nucleating agent for isotactic polypropylene and its influence on the polymorphic transitions", J. Appl. Polym. Sci., 86[3], 531-539 (2002)

9. C. Marco, C. Blancas, artículo en preparación

10. G. Ellis, M. Gómez, C. Marco. "Practical considerations in the study of mainchain thermotropic liquid-crystalline polymers by vibrational microscopy". Analusis, Eur. J. Anal. Chem. 28[1], 22-29 (2000)

11. Perkin-Elmer. "Spectrum spotlight 300 IR Imaging System", Información de Producto, D-6545A (2002)

12. P. Dumas, G.L.Carr, G.P. Williams. "Enhancing the lateral resolution in infrared microspectrometry by using synchrotron radiation: applications and perspectives". Analusis, Eur. J. Anal. Chem. 28[1], 68-74 (2000)

13. P. Dumas. "MIRAGE", http://www.lure.u-psud.fr/Experiences/SACO/ SA5/ Anglaise/intro.htm

14. Renishaw. "RM1000B entry-level Raman microscope", Información de Producto, SPE-APP-0013 (2001)

15. G. Ellis, "Studies on the heterogeneity of polymeric systems by vibrational microscopy" Macromol. Symp. 184[1], 37-47 (2002)

16. G. Ellis, C. Marco, M.A. Gómez, E.P. Collar and J.M. García-Martínez, “The study of heterogeneous polymer systems by synchrotron infrared microscopy", Synchrotron Radiation in Polymer Science II, Sheffield (UK), septiembre 2002. J. Macromol. Sci. Phys., aceptado.

Recibido: 1.2.03

Aceptado: 30.11 .03 